\title{
DNA Damage Induced by Alkylating Agents and Repair Pathways
}

\author{
Natsuko Kondo, ${ }^{1}$ Akihisa Takahashi, ${ }^{2}$ Koji Ono, ${ }^{1}$ and Takeo Ohnishi ${ }^{3}$ \\ ${ }^{1}$ Particle Radiation Oncology Research Center, Research Reactor Institute, Kyoto University, Kumatori-cho, Sennan-gun, \\ Osaka 590-0494, Japan \\ ${ }^{2}$ Department of Biology, School of Medicine, Nara Medical University, 840 Shijo-cho, Kashihara, Nara 634-8521, Japan \\ ${ }^{3}$ Department of Radiation Oncology, School of Medicine, Nara Medical University, 840 Shijo-cho, Kashihara, Nara 634-8521, Japan
}

Correspondence should be addressed to Akihisa Takahashi, atakahas@naramed-u.ac.jp

Received 8 June 2010; Revised 26 August 2010; Accepted 12 October 2010

Academic Editor: Ashis Basu

Copyright (c) 2010 Natsuko Kondo et al. This is an open access article distributed under the Creative Commons Attribution License, which permits unrestricted use, distribution, and reproduction in any medium, provided the original work is properly cited.

\begin{abstract}
The cytotoxic effects of alkylating agents are strongly attenuated by cellular DNA repair processes, necessitating a clear understanding of the repair mechanisms. Simple methylating agents form adducts at $N$ - and $O$-atoms. $N$-methylations are removed by base excision repair, AlkB homologues, or nucleotide excision repair (NER). $O^{6}$-methylguanine (MeG), which can eventually become cytotoxic and mutagenic, is repaired by $O^{6}$-methylguanine-DNA methyltransferase, and $O^{6} \mathrm{MeG}$ :T mispairs are recognized by the mismatch repair system (MMR). MMR cannot repair the $O^{6} \mathrm{MeG} / \mathrm{T}$ mispairs, which eventually lead to double-strand breaks. Bifunctional alkylating agents form interstrand cross-links (ICLs) which are more complex and highly cytotoxic. ICLs are repaired by complex of NER factors (e.g., endnuclease xeroderma pigmentosum complementation group Fexcision repair cross-complementing rodent repair deficiency complementation group 1), Fanconi anemia repair, and homologous recombination. A detailed understanding of how cells cope with DNA damage caused by alkylating agents is therefore potentially useful in clinical medicine.
\end{abstract}

\section{Introduction}

Alkylating drugs are the oldest class of anticancer drugs still commonly used; they play an important role in the treatment of several types of cancers [1]. Most alkylating drugs are monofunctional methylating agents (e.g., temozolomide [TMZ], $N$-methyl- $N^{\prime}$-nitro- $N$-nitrosoguanidine [MNNG], and dacarbazine), bifunctional alkylating agents such as nitrogen mustards (e.g., chlorambucil and cyclophosphamide), or chloroethylating agents (e.g., nimustine [ACNU], carmustine [BCNU], lomustine [CCNU], and fotemustine).

Simple methylating agents form adducts at the $\mathrm{N}$ - and $\mathrm{O}$ atoms in DNA bases. $N$-methylation adducts comprise more than $80 \%$ of methylated bases. These alkyl DNA base adducts exhibit different stabilities. For example, $N^{7}$-methylguanine $\left(N^{7} \mathrm{MeG}\right)$ is the most stable $N$-methylation adduct in vitro with a half-life $\left(t_{1 / 2}\right)$ no longer than $80 \mathrm{~h}$ [2]. Although $O^{6}$-methylguanine $\left(O^{6} \mathrm{MeG}\right)$ accounts for only $0.3 \%$ (for methyl methanesulfonate) to $8 \%$ (for methylnitrosourea) of the total DNA methyl adducts, it is stable and persists in the absence of the DNA repair protein $O^{6}$-methylguanineDNA methyltransferase (MGMT) [3-5]. $O^{4}$-methylthymine $\left(O^{4} \mathrm{MeT}\right)$ is produced at a much lower level $(<0.4 \%)$ [2], and its mutagenicity and cytotoxicity are unclear. In general, $O$-alkylations (e.g., $O^{6}$ alkylG and $O^{4}$ alkylT) are highly mutagenic and genotoxic, whereas $\mathrm{N}$-alkylations (e.g., $N^{3}$ alkylA and $N^{1}$ alkylA) are cytotoxic, but less mutagenic [6-9]. The primary products of methylating agents, $\mathrm{N}$ alkylated purines, are efficiently removed by base excision repair (BER) or human AlkB homologues (hABH). BER repairs $N^{7} \mathrm{MeG}, N^{3} \mathrm{MeA}$, and $N^{3} \mathrm{MeG}$, whereas hABH repairs $N^{1} \mathrm{MeA}, N^{3} \mathrm{MeC}, N^{3} \mathrm{MeT}$, and $N^{1} \mathrm{MeG}[10]$.

One-step repair of $O^{6} \mathrm{MeG}$ involves transferring the alkyl group from the oxygen in the guanine to a cysteine residue in the catalytic pocket of MGMT [10]. Nucleotide excision repair (NER) is an elaborate repair system that removes bulky lesions from DNA in 27-nt to 29-nt oligomers. Because it is also capable of removing nonbulky lesions such as apurinic/apyrimidinic sites and $O^{6} \mathrm{MeG}$ residues, NER plays 
a backup role for other repair systems [11]. Mismatch repair (MMR) is also important in the repair of $O^{6} \mathrm{MeG}$. If it is left unrepaired, replication over the $O^{6} \mathrm{MeG}$ results in an $\mathrm{O}^{6} \mathrm{MeG}: \mathrm{T}$ mismatch or $\mathrm{O}^{6} \mathrm{MeG}: \mathrm{C}$ ambiguous pair [12]. In the next round of replication, the $O^{6} \mathrm{MeG}$ : $\mathrm{T}$ becomes an $\mathrm{A}: \mathrm{T}$ transition mutation, or the $O^{6} \mathrm{MeG}$ : $\mathrm{C}$ is replicated again as an $\mathrm{O}^{6} \mathrm{MeG}$ :C pair or becomes an $\mathrm{O}^{6} \mathrm{MeG}$ :T mismatch [13]. The $\mathrm{O}^{6} \mathrm{MeG}: \mathrm{T}$ or $\mathrm{O}^{6} \mathrm{MeG}: \mathrm{C}$ is recognized by the MutS $\alpha$ complex (hMSH2 and hMSH6), which initiates MMR to create a gapped duplex by incision of the newly replicated strand [13]. If $O^{6} \mathrm{MeG}$ remains in the template, a futile repair loop can eventually result in highly toxic double-strand breaks (DSBs), which are intermediates in apoptotic and DSB repair pathways [13]. Accordingly, DSB repair pathways are activated by methylating agents $[14,15]$.

Bifunctional alkylating agents, such as chlorambucil or BCNU, are commonly used anticancer drugs. DNA lesions produced by these agents require complex repair mechanisms. The primary chloroethyl adducts at $O^{6} \mathrm{G}$ are repaired by MGMT, but the secondary interstrand cross-links (ICLs) require NER factors (e.g., endnuclease xeroderma pigmentosum complementation group F-excision repair crosscomplementing rodent repair deficiency complementation group 1 (XPF-ERCC1)) for incision, Fanconi anemia (FA) repair, and homologous recombination (HR) for complete repair [16].

This paper will focus on the repair pathways for $O^{6} \mathrm{MeG}$ generated by methylating agents and those for ICLs generated by bifunctional alkylating agents. We will also briefly discuss other alkylation damage defense and processing functions (hABH and BER).

\section{DNA Repair Mechanisms for DNA Damage Induced by Methylating Agents}

2.1. MGMT (Figure 1(a)). MGMT repairs $O^{6}$-alkylation adducts but irreversibly inactivates MGMT itself in the process. In the absence of active MGMT, $\mathrm{O}^{6} \mathrm{MeG}$ forms $\mathrm{O}^{6} \mathrm{MeG} / \mathrm{T}$ mismatches during replication. Early studies demonstrated that MGMT-deficient cells unable to repair $\mathrm{O}^{6} \mathrm{MeG}$ damage were more sensitive to the effects of methylating agents than normal cells expressing MGMT [17]. This observation has been utilized experimentally and clinically to target cells with an MGMT inhibitor, the $O^{6} \mathrm{MeG}$ analogue $O^{6}$ benzylG [18]. However, in some tumors, $p 53$ dysfunction suppresses MGMT expression [19, 20] or hypermethylation of the MGMT promoter results in gene silencing [21]. The low basal MGMT activity makes these cells less vulnerable to the effects of $O^{6}$ benzylG. Kaina et al. reported that about $5 \%$ of all solid tumors assayed in their laboratory were completely deficient in MGMT [10]. In particular, $17 \%$ to $30 \%$ of gliomas lack MGMT $[22,23]$. Because drug efficacy is likely to be limited if only MGMT is targeted in these tumors, new molecular targets are being sought.

2.2. MMR (Figure 1(b)). The cytotoxicity of monofunctional alkylating agents requires a functional MMR in the target cells. In fact, mammalian cells proficient in MMR are generally about 100 -fold more sensitive to alkylating agents than their MMR-deficient counterparts [24, 25]. In MMRdeficient cells, DNA damage accumulates but does not trigger cell death. Thus, resistance to these cytotoxic agents is associated with loss of MMR activity, particularly in the absence of MGMT $[26,27]$. The mechanism of action of monofunctional alkylating agents has been studied in cell lines and mouse models; results indicate that replication over unrepaired $\mathrm{O}^{6} \mathrm{MeG}: \mathrm{C}$ results in an $\mathrm{O}^{6} \mathrm{MeG}$ : $\mathrm{T}$ mismatch (or possibly an $\mathrm{O}^{6} \mathrm{MeG}: \mathrm{C}$ ambiguous pair). In the next round of replication, an $\mathrm{O}^{6} \mathrm{MeG}: \mathrm{T}$ mismatch becomes an A:T transition mutation. An $O^{6} \mathrm{MeG}: \mathrm{T}$ or $O^{6} \mathrm{MeG}$ :C pair is recognized by the MutS $\alpha$ complex, which initiates MMR. MMR creates a gapped duplex after incision of the newly replicated strand. The mere presence of $\mathrm{MeG}$ in the genomic DNA of MMR-proficient cells is not cytotoxic, even if the cells are allowed to undergo a round of replication during which MeG:C and MeG:T pairs form. To activate the $\mathrm{G}_{2} / \mathrm{M}$ DNA damage checkpoint, these mispairs must be recognized and processed. Cells treated with $\mathrm{MNNG}$ are not arrested in the first $\mathrm{G}_{2} / \mathrm{M}$ checkpoint, but $\mathrm{G}_{2} / \mathrm{M}$ arrest is commonly observed in the second cell cycle [28].

2.3. DSB Repair (Figure 1(c)). Although alkylating agents do not directly induce DSBs, DSBs are detected in wild-type cells and other cell culture systems after the processing of DNA lesions induced by alkylating agents $[14,15,29,30]$. DSBs lead to cell death; therefore, cells defective in DSB repair are thought to be more sensitive to alkylating agents. Consistent with this hypothesis, studies have reported that DSB repair pathways are involved in the repair of DNA damage induced by alkylating agents $[14,15,29]$.

DSBs are repaired through the HR and nonhomologous end joining (NHEJ) pathways [31]. In human cells, HR proteins include members of the MRN complex, which consist of meiotic recombination 11 (MRE11)/radiationsensitive mutant 50 (Rad50)/Nijmegen breakage syndrome 1 (NBS1) as well as Rad51, the Rad51 paralogs (Rad51B, Rad51C, Rad51D, X-ray repair cross-complementing group 2 (XRCC2), and XRCC3), Rad54, and Rad54B [31]. Proteins involved in the NHEJ pathway include Ku70/80, the DNAdependent protein kinase catalytic subunit (DNA-PKcs), ligase IV (Lig4), XRCC4 and Artemis [31].

$\mathrm{HR}$, which is a generally error-free pathway, uses DNA homology to direct DNA repair; an undamaged chromatid serves as template for the repair of a broken sister chromatid. The products of the breast cancer susceptibility genes, BRCA1 and BRCA2 (also known as FA complementation group D1 or FANCD1), are also involved in the HR pathway [32].

HR is required for MNNG-treated cells to transition into the second cell cycle. Most mammalian cells that undergo cell cycle arrest after the second S phase die; however, the surviving cells show a high frequency of sister chromatid exchanges (SCEs), indicative of DSB repair at collapsed replication forks [33]. Roos et al. reported that BRCA2/XRCC2-dependent $\mathrm{HR}$, but not NHEJ, protects against $\mathrm{O}^{6} \mathrm{MeG}$-triggered DSBs and chromosomal aberrations, leading to SCEs [14]. 
(c) DSB repair

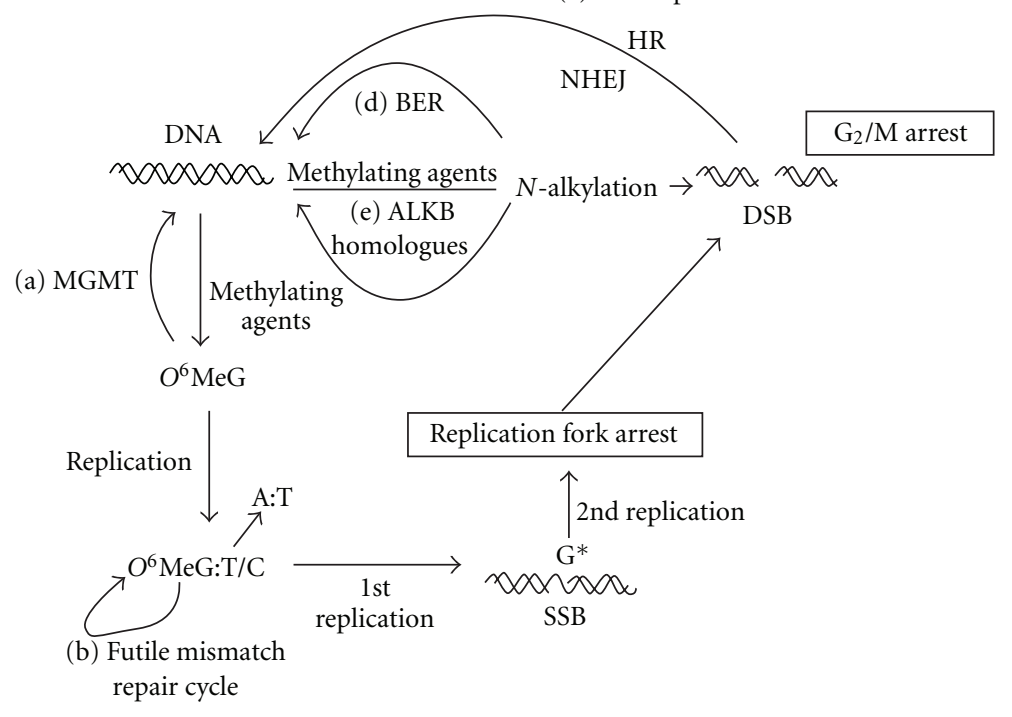

Figure 1: Pathways for DNA damage induced by methylating agents. (a) $O^{6}$-methylguanine-DNA methyltransferase (MGMT) removes the methyl adduct from $O^{6} \mathrm{MeG}$ in one step. If left unrepaired, $O^{6} \mathrm{MeG}$ :C ambiguous pairs or $O^{6} \mathrm{MeG}$ : $\mathrm{T}$ mismatch pairs can form during replication. In the next round of replication, $O^{6} \mathrm{MeG}: \mathrm{T}$ pairs can become A:T transition mutations. (b) $O^{6} \mathrm{MeG}: \mathrm{T}$ and $O^{6} \mathrm{MeG}: \mathrm{C}$ pairs are recognized by the mismatch repair (MMR) system, which creates a single-strand break (SSB), cause replication arrest, and finally leads to a double-strand break (DSB). $\mathrm{O}^{6} \mathrm{MeG}: \mathrm{T} / \mathrm{C}$ does not induce cell cycle arrest at the first $\mathrm{G}_{2} / \mathrm{M}_{\mathrm{DNA}}$ damage checkpoint, but $\mathrm{G}_{2} / \mathrm{M}$ arrest is commonly observed in the second cell cycle. (c) Homologous recombination (HR) and nonhomologous end joining (NHEJ) may play a role in the repair of DSBs. $N$-alkylations are repaired by either (d) base excision repair (BER), or (e) AlkB homologues, and if not repaired, DSBs occur.

NHEJ, which is the simplest way to repair a DSB, involves the religation of broken DNA ends without a template; this type of repair does not preserve the original genetic information. NHEJ eliminates DSBs during the $G_{1}$ phase of the cell cycle, when the lack of sister chromatids prevents HR [34].

Results of a clonogenic survival study showed that Lig4 plays a more important role in the repair of TMZ-induced DSBs than XRCC2 or Rad54 [15]. DSBs, which may arise from adducts other than $O^{6} \mathrm{MeG}$, such as TMZ-induced $\mathrm{N}$ methylpurines, are repaired within $24 \mathrm{~h}$ in Lig4-proficient cells. In contrast, up to $80 \%$ of the DSBs in $\mathrm{Lig}^{-/-}$cells were not repaired [15]. In A172 glioblastoma cells, siRNA silencing of Lig4 increased cellular sensitivity to TMZ. After TMZ treatments, A172 cells with silenced Lig4 exhibited a $62.5 \%$ decrease in survival compared with control A172 cells; thus, modulating Lig4 activity may enhance tumor sensitivity to TMZ [15].

2.4. BER (Figure 1(d)). The alkylation adducts $N^{7} \mathrm{MeG}$, $N^{3} \mathrm{MeA}$, and $N^{3} \mathrm{MeG}$ are repaired by the BER system, the main DNA repair system in mammalian cells used to eliminate small DNA base lesions [16]. Damaged bases are removed by a lesion-specific DNA glycosylase, in this case alkyladenine DNA glycosylase (Aag). The resulting abasic site is recognized by an apurinic/apyrimidinic endonuclease, APE1, which incises the damaged strand, leaving $3^{\prime}-\mathrm{OH}$ and $5^{\prime}$ deoxyribose phosphate $\left(5^{\prime}-\mathrm{dRP}\right)$ groups at the margins. A DNA polymerase $\beta$ - (pol- $\beta$-)mediated DNA synthesis step fills the single nucleotide gap $[35,36]$ and removes the cytotoxic $5^{\prime}$-dRP group $[37,38]$. Alternatively, DNA polymerase $\lambda$ (pol- $\lambda$ ) or DNA polymerase- $\iota$ (pol- $\iota$ ), both of which possess $5^{\prime}$-dRP lyase activity, may participate in BER to remove this toxic repair intermediate [39-41]. Finally, DNA ligase I, or a complex of DNA ligase III and XRCC1, conducts the final, nick-sealing step in the pathway [42].

In the absence of pol- $\beta$, cells cannot repair the BER intermediate $5^{\prime} \mathrm{dRP}$ and are thus hypersensitive to the alkylating agent methyl methanesulfonate $[37,38,43,44]$. For example, fibroblasts from a pol- $\beta$-null mutant mouse are highly sensitive to monofunctional alkylating agents, but not to BCNU [45]. Similarly, RNA interference-mediated pol- $\beta$ suppression boosts TMZ efficacy, although a deficiency in pol- $\iota$ or pol- $\lambda$ does not increase TMZ-mediated cytotoxicity [46]. Furthermore, loss of pol- $\beta$ coupled with TMZ treatment triggers H2AX phosphorylation, indicating activation of the DNA damage response pathway by unrepaired lesions [46]. H2AX is a histone protein that is rapidly phosphorylated on Ser139 $(\gamma \mathrm{H} 2 \mathrm{AX})$ when DNA breaks are introduced in mammalian cells following external damage and replication fork collapse $[47,48]$. Poly(ADP-ribose) polymerase-1 (PARP-1) is activated by strand breaks and participates in gap sealing with DNA ligase III and XRCC1, but deficiencies in the subsequent steps of BER increase sensitivity to alkylating agents. Inhibition of PARP-1 by the inhibitor AG14361 restores sensitivity to TMZ in MMRdeficient cells that have lost killing sensitivity to $O^{6} \mathrm{MeG}$ via the MGMT/MMR pathway [49]. The combination of TMZ with PARP-1 inhibitors is currently under investigation in several Phase I-II clinical trials. 
2.5. Direct Reversal of Alkylation Damage by AlkB Homologues (Figure 1(e)). The E. coli protein AlkB is an oxidative DNA demethylase that repairs the cytotoxic lesions $N^{1} \mathrm{MeA}$ and $N^{3} \mathrm{MeC}$. A detailed mapping of the human genome has identified eight hABH homologues. $\mathrm{ABH} 2$ and $\mathrm{ABH} 3$ belong to the alpha-ketoglutarate- and $\mathrm{Fe}(\mathrm{II})$-dependent dioxygenase superfamily. These proteins repair $N^{1} \mathrm{MeA}$, $N^{3} \mathrm{MeC}, N^{3} \mathrm{MeT}$, and $N^{1} \mathrm{MeG}$ by oxidative demethylation [50, 51]. Although hABH2 preferentially repairs doublestranded DNA, hABH3 acts more efficiently on singlestranded nucleic acids. Accordingly, hABH2 relocates to replication foci during $\mathrm{S}$-phase, which suggested that hABH2 repairs DNA close to replication forks, whereas hABH3 maintains nuclear single-stranded DNA and RNA, potentially targeting genes undergoing transcription.

\section{Repair of Cross-links Induced by Bifunctional Alkylating Agents}

Bifunctional alkylating agents (e.g., nitrogen mustards (melphalan, chlorambucil, cyclophosphamide, and ifosfamide) and chloroethylnitrosoureas (BCNU and CCNU)) possess two reactive sites. These agents cross-link DNA with proteins or, alternatively, cross-link two DNA bases within the same DNA strand (intrastrand cross-links) or on opposite DNA strands (ICLs). ICLs, which block replication forks, are the most serious cytotoxic lesions produced by most bifunctional drugs. Accordingly, the extent of ICLs correlates well with the cytotoxicity of nitrogen mustard drugs [52].

Nitrogen mustards form $N^{7} G: N^{7} G$ cross-links, and chloroethylnitrosoureas form $N^{1} G: N^{3} C$ cross-links [53]. The chloroethylated $O^{6} \mathrm{G}$ of the $N^{1} G: N^{3} C$ cross-link can be repaired by MGMT; however, this adduct is unstable and undergoes intramolecular rearrangement producing an intermediary $N^{1}-O^{6}$-ethanoG. The $N^{1}-O^{6}$-ethanoG adduct may react with cytosine in the complementary strand to yield a highly toxic ICL between position 1 in the guanine residue and position 3 in the cytosine residue (1-(3-cytosinyl)-2-(1guanosinyl)-ethane) [53].

ICL repair mechanisms are complex; therefore, they are only briefly summarized here. An ICL represents a formidable block to the DNA replication machinery and is unique in requiring a combination of FA repair, NER, translesion synthesis (TLS), and HR repair for efficient repair [54]. Although the FA pathway was initially characterized in terms of DNA cross-link repair, this pathway is also involved in homologous recombination and resolution of the replication arrest $[55,56]$. Thirteen FA genes have been identified [54], although the precise function of many of these FA proteins is unclear. The FA core complex, which consists of eight FA proteins, is activated by DNA damage. Specifically, the FA proteins FANCM and FANCA Associating Polypeptide 24 form a heterodimer that binds DNA $[57,58]$ and appears to be involved in sensing DNA replication forks blocked at cross-links. The NER proteins ERCC1 and XPF make incisions on either side of the crosslink to generate a gap. The gap is then filled by translesion synthesis (TLS) polymerases $\zeta$ (Rev3 and Rev7 subunits) and Rev1 (part of the Rev3-Rev7 complex [59]). The FA core complex monoubiquitinates FANCD2 and its paralog FANCI, and the ubiquitinated FANCD2 then interacts with FANCD1 to promote HR [54].

Incision at the ICL could occur before or after lesion bypass, leaving a DSB subject to $\mathrm{HR}$ or NHEJ [60]. As expected, XRCC2 and Rad54 are involved in the repair of ACNU-induced DSBs, but surprisingly Lig4 plays the most important role in this process [29]. In Lig4 ${ }^{-/-}$cells, levels of phosphorylated histone $\gamma \mathrm{H} 2 \mathrm{AX}$ increased more than 4 -fold at $12 \mathrm{~h}$ and 6 -fold at $24 \mathrm{~h}$ after ACNU treatment compared to its initial levels. In contrast, $\gamma \mathrm{H} 2 \mathrm{AX}$ levels were not markedly altered by ACNU in normal cells. In addition, ACNU treatment markedly reduced the colony-forming ability of A172 glioblastoma cells transfected with siRNA against Lig4 or XRCC2 compared to controls [29]. However, Lig4 siRNA rendered cells more sensitive to the effects of ACNU than did XRCC2 siRNA [29]. These data suggest NHEJ may also be involved in removing DSBs formed by unrepaired ICLs.

\section{Conclusion}

DNA repair pathways attenuate the therapeutic effects of alkylating agents; therefore, characterization of the repair pathways is essential for developing new treatments. For example, MGMT promoter hypermethylation results in gene silencing and therefore decreased MGMT activity; therefore, MGMT promoter hypermethylation may be a useful way to enhance the therapeutic efficacy of TMZ [61, 62].

Currently, clinical trials are testing DNA repair inhibitors that target PARP, BER, or MGMT in combination with alkylating agents [63]. In the case of $O^{6}$ benzylG, a phase I clinical trial has defined the maximum tolerated dose of a single dose of TMZ when combined with $O^{6}$ benzylG and has determined the dose of $O^{6}$ benzylG that depletes tumor MGMT activity for $48 \mathrm{~h}$ [64]. In addition, when combined with cytotoxic chemotherapy, myelosuppression appears to be significantly enhanced by $O^{6}$ benzylG, significantly reducing the required doses of alkylating agents [65]. The success of such approaches will depend on selective targeting of the tumor. Locoregional chemotherapy has recently been shown to improve the survival of glioma patients [66]. Therefore, combining a locoregional delivery system with the simultaneous downregulation of DNA repair pathways may decrease the amount of alkylating agent needed for chemotherapy, thereby reducing the severe side effects. In addition, new inhibitors against specific repair proteins, such as pol- $\beta$, BRCA2, or Lig4, should be developed because resistance against currently available inhibitors may develop.

\section{Acknowledgments}

This work was supported by Grants-in-Aid for Scientific Research from the Ministry of Education, Culture, Sports, Science and Technology of Japan. This work was also funded in part by a grant from the Central Research Institute of the Electric Power Industry in Japan and by a grant for Exploratory Research for Space Utilization from the Japan Space Forum. 


\section{References}

[1] S. G. Chaney and A. Sancar, "DNA repair: enzymatic mechanisms and relevance to drug response," Journal of the National Cancer Institute, vol. 88, no. 19, pp. 1346-1360, 1996.

[2] D. T. Beranek, "Distribution of methyl and ethyl adducts following alkylation with monofunctional alkylating agents," Mutation Research, vol. 231, no. 1, pp. 11-30, 1990.

[3] R. Goth and M. F. Rajewsky, "Persistence of $O^{6}$ ethylguanine in rat brain DNA: correlation with nervous system specific carcinogenesis by ethylnitrosourea," Proceedings of the National Academy of Sciences of the United States of America, vol. 71, no. 3, pp. 639-643, 1974.

[4] R. Goth-Goldstein, "Inability of Chinese hamster ovary cells to excise $O^{6}$-alkylguanine," Cancer Research, vol. 40, no. 7, pp. 2623-2624, 1980.

[5] B. Kaina, A. A. van Zeeland, A. de Groot, and A. T. Natarajan, "DNA repair and chromosomal stability in the alkylating agent-hypersensitive Chinese hamster cell line 27-1," Mutation Research, vol. 243, no. 3, pp. 219-224, 1990.

[6] G. P. Margison, M. F. Santibáñez-Koref, and A. C. Povey, "Mechanisms of carcinogenicity/chemotherapy by $\mathrm{O}^{6}$ methylguanine," Mutagenesis, vol. 17, no. 6, pp. 483-487, 2002.

[7] M. Christmann, M. T. Tomicic, W. P. Roos, and B. Kaina, "Mechanisms of human DNA repair: an update," Toxicology, vol. 193, no. 1-2, pp. 3-34, 2003.

[8] M. D. Wyatt, J. M. Allan, A. Y. Lau, T. E. Ellenberger, and L. D. Samson, "3-Methyladenine DNA glycosylases: structure, function, and biological importance," BioEssays, vol. 21, no. 8, pp. 668-676, 1999.

[9] H. E. Krokan, R. Standal, and G. Slupphaug, "DNA glycosylases in the base excision repair of DNA," Biochemical Journal, vol. 325, no. 1, pp. 1-16, 1997.

[10] B. Kaina, M. Christmann, S. Naumann, and W. P. Roos, "MGMT: key node in the battle against genotoxicity, carcinogenicity and apoptosis induced by alkylating agents," DNA Repair, vol. 6, no. 8, pp. 1079-1099, 2007.

[11] A. Sancar, "Excision repair in mammalian cells," The Journal of Biological Chemistry, vol. 270, no. 27, pp. 15915-15918, 1995.

[12] H. T. Chen, A. Bhandoola, M. J. Difilippantonio et al., "Response to RAG-mediated V(D)J cleavage by NBS1 and $\gamma$ H2AX," Science, vol. 290, no. 5498, pp. 1962-1964, 2000.

[13] G. P. Margison and M. F. Santibáñez-Koref, " $\mathrm{O}^{6}$-alkylguanineDNA alkyltransferase: role in carcinogenesis and chemotherapy," BioEssays, vol. 24, no. 3, pp. 255-266, 2002.

[14] W. P. Roos, T. Nikolova, S. Quiros et al., "Brca2/Xrcc2 dependent HR, but not NHEJ, is required for protection against $O^{6}$ methylguanine triggered apoptosis, DSBs and chromosomal aberrations by a process leading to SCEs," DNA Repair, vol. 8, no. 1, pp. 72-86, 2009.

[15] N. Kondo, A. Takahashi, E. Mori et al., "DNA ligase IV as a new molecular target for temozolomide," Biochemical and Biophysical Research Communications, vol. 387, no. 4, pp. 656660, 2009.

[16] F. Drabløs, E. Feyzi, P. A. Aas et al., "Alkylation damage in DNA and RNA-repair mechanisms and medical significance," DNA Repair, vol. 3, no. 11, pp. 1389-1407, 2004.

[17] R. S. Day III, C. H. J. Ziolkowski, and D. A. Scudiero, "Defective repair of alkylated DNA by human tumour and SV40-transformed human cell strains," Nature, vol. 288, no. 5792, pp. 724-727, 1980.
[18] N. Hosoya and K. Miyagawa, "Clinical importance of DNA repair inhibitors in cancer therapy," Memo-Magazine of European Medical Oncology, vol. 2, no. 1, pp. 9-14, 2009.

[19] M. D. Blough, M. C. Zlatescu, and J. G. Cairncross, " $\mathrm{O}^{6}$ methylguanine-DNA methyltransferase regulation by p53 in astrocytic cells," Cancer Research, vol. 67, no. 2, pp. 580-584, 2007.

[20] S. J. Russell, Y.-W. Ye, P. G. Waber, M. Shuford, S. C. Schold Jr., and P. D. Nisen, "p53 Mutations, $\mathrm{O}^{6}$-alkylguanine DNA alkyltransferase activity, and sensitivity to procarbazine in human brain tumors," Cancer, vol. 75, no. 6, pp. 1339-1342, 1995.

[21] M. Esteller, S. R. Hamilton, P. C. Burger, S. B. Baylin, and J. G. Herman, "Inactivation of the DNA repair gene $O^{6}$ methylguanine-DNA methyltransferase by promoter hypermethylation is a common event in primary human neoplasia," Cancer Research, vol. 59, no. 4, pp. 793-797, 1999.

[22] I. Preuss, I. Eberhagen, S. Haas et al., " $O^{6}$-methylguanineDNA methyltransferase activity in breast and brain tumors," International Journal of Cancer, vol. 61, no. 3, pp. 321-326, 1995.

[23] N. P. Lees, K. L. Harrison, E. Hill, C. Nicholas Hall, A. C. Povey, and G. P. Margison, "Heterogeneity of $\mathrm{O}^{6}$-alkylguanine-DNA alkyltransferase activity in colorectal cancer: implications for treatment," Oncology, vol. 63, no. 4, pp. 393-397, 2002.

[24] P. Karran, "Mechanisms of tolerance to DNA damaging therapeutic drugs," Carcinogenesis, vol. 22, no. 12, pp. 19311937, 2001.

[25] L. Stojic, R. Brun, and J. Jiricny, "Mismatch repair and DNA damage signalling," DNA Repair, vol. 3, no. 8-9, pp. 10911101, 2004.

[26] P. Branch, G. Aquilina, M. Bignami, and P. Karran, "Defective mismatch binding and a mutator phenotype in cells tolerant to DNA damage," Nature, vol. 362, no. 6421, pp. 652-654, 1993.

[27] A. Kat, W. G. Thilly, W.-H. Fang, M. J. Longley, G.-M. Li, and P. Modrich, "An alkylation-tolerant, mutator human cell line is deficient in strand- specific mismatch repair," Proceedings of the National Academy of Sciences of the United States of America, vol. 90, no. 14, pp. 6424-6428, 1993.

[28] S. Quiros, W. P. Roos, and B. Kaina, "Processing of $\mathrm{O}^{6}$ methylguanine into DNA double-strand breaks requires two rounds of replication whereas apoptosis is also induced in subsequent cell cycles," Cell Cycle, vol. 9, no. 1, pp. 168-178, 2010.

[29] N. Kondo, A. Takahashi, E. Mori et al., "DNA ligase IV is a potential molecular target in ACNU sensitivity," Cancer Science, vol. 101, no. 8, pp. 1881-1885, 2010.

[30] S. C. Naumann, W. P. Roos, E. Jöst et al., "Temozolomideand fotemustine-induced apoptosis in human malignant melanoma cells: response related to MGMT, MMR, DSBs, and p53," British Journal of Cancer, vol. 100, no. 2, pp. 322-333, 2009.

[31] T. Ohnishi, E. Mori, and A. Takahashi, "DNA doublestrand breaks: their production, recognition, and repair in eukaryotes," Mutation Research, vol. 669, no. 1-2, pp. 8-12, 2009.

[32] A. A. Davies, J. Y. Masson, M. J. McIlwraith et al., "Role of BRCA2 in control of the RAD51 recombination and DNA repair protein," Molecular Cell, vol. 7, no. 2, pp. 273-282, 2001.

[33] N. Mojas, M. Lopes, and J. Jiricny, "Mismatch repairdependent processing of methylation damage gives rise to 
persistent single-stranded gaps in newly replicated DNA," Genes and Development, vol. 21, no. 24, pp. 3342-3355, 2007.

[34] T. Helleday, J. Lo, D. C. van Gent, and B. P. Engelward, "DNA double-strand break repair: from mechanistic understanding to cancer treatment," DNA Repair, vol. 6, no. 7, pp. 923-935, 2007.

[35] R. W. Sobol and S. H. Wilson, "Mammalian DNA $\beta$ polymerase in base excision repair of alkylation damage," Progress in Nucleic Acid Research and Molecular Biology, vol. 68, pp. 57-74, 2001.

[36] S. H. Wilson, R. W. Sobol, W. A. Beard, J. K. Horton, R. Prasad, and B. J. Vande Berg, "DNA polymerase $\beta$ and mammalian base excision repair," Cold Spring Harbor Symposia on Quantitative Biology, vol. 65, pp. 143-155, 2000.

[37] R. W. Sobol, J. K. Horton, R. Kühn et al., "Requirement of mammalian DNA polymerase- $\beta$ in base-excision repair," Nature, vol. 379, no. 6561, pp. 183-186, 1996.

[38] R. W. Sobol, R. Prasad, A. Evenski et al., "The lyase activity of the DNA repair protein $\beta$-polymerase protects from DNAdamage-induced cytotoxicity," Nature, vol. 405, no. 6788, pp. 807-810, 2000.

[39] R. Prasad, K. Bebenek, E. Hou et al., "Localization of the deoxyribose phosphate lyase active site in human DNA polymerase $\beta$ by controlled proteolysis," The Journal of Biological Chemistry, vol. 278, no. 32, pp. 29649-29654, 2003.

[40] K. Bebenek, A. Tissier, E. G. Frank et al., " 5 '-deoxyribose phosphate lyase activity of human DNA polymerase L in vitro," Science, vol. 291, no. 5511, pp. 2156-2159, 2001.

[41] M. García-Díaz, K. Bebenek, T. A. Kunkel, and L. Blanco, "Identification of an intrinsic 5'-deoxyribose-5-phosphate lyase activity in human DNA polymerase $\lambda$ : a possible role in base excision repair," The Journal of Biological Chemistry, vol. 276, no. 37, pp. 34659-34663, 2001.

[42] T. Lindahl and R. D. Wood, "Quality control by DNA repair," Science, vol. 286, no. 5446, pp. 1897-1905, 1999.

[43] R. W. Sobolt, M. Kartalou, K. H. Almeida et al., "Base excision repair intermediates induce p53-independent cytotoxic and genotoxic responses," The Journal of Biological Chemistry, vol. 278, no. 41, pp. 39951-39959, 2003.

[44] R. W. Sobol, D. E. Watson, J. Nakamura et al., "Mutations associated with base excision repair deficiency and methylationinduced genotoxic stress," Proceedings of the National Academy of Sciences of the United States of America, vol. 99, no. 10, pp. 6860-6865, 2002.

[45] J. K. Horton, D. F. Joyce-Gray, B. F. Pachkowski, J. A. Swenberg, and S. H. Wilson, "Hypersensitivity of DNA polymerase $\beta$ null mouse fibroblasts reflects accumulation of cytotoxic repair intermediates from site-specific alkyl DNA lesions," DNA Repair, vol. 2, no. 1, pp. 27-48, 2003.

[46] R. N. Trivedi, K. H. Almeida, J. L. Fornsaglio, S. Schamus, and R. W. Sobol, "The role of base excision repair in the sensitivity and resistance to temozolomide-mediated cell death," Cancer Research, vol. 65, no. 14, pp. 6394-6400, 2005.

[47] E. P. Rogakou, D. R. Pilch, A. H. Orr, V. S. Ivanova, and W. M. Bonner, "DNA double-stranded breaks induce histone H2AX phosphorylation on serine 139," The Journal of Biological Chemistry, vol. 273, no. 10, pp. 5858-5868, 1998.

[48] I. M. Ward and J. Chen, "Histone H2AX is phosphorylated in an ATR-dependent manner in response to replicational stress," The Journal of Biological Chemistry, vol. 276, no. 51, pp. 47759-47762, 2001.
[49] N. J. Curtin, L.-Z. Wang, A. Yiakouvaki et al., "Novel poly(ADP-ribose) polymerase-1 inhibitor, AG14361, restores rensitivity to temozolomide in mismatch repair-deficient cells," Clinical Cancer Research, vol. 10, no. 3, pp. 881-889, 2004.

[50] T. Duncan, S. C. Trewick, P. Koivisto, P. A. Bates, T. Lindahl, and B. Sedgwick, "Reversal of DNA alkylation damage by two human dioxygenases," Proceedings of the National Academy of Sciences of the United States of America, vol. 99, no. 26, pp. 16660-16665, 2002.

[51] P. A. Aas, M. Otterlei, P. O. Falnes et al., "Human and bacterial oxidative demethylases repair alkylation damage in both RNA and DNA," Nature, vol. 421, no. 6925, pp. 859-863, 2003.

[52] P. M. O'Connor and K. W. Kohn, "Comparative pharmacokinetics of DNA lesion formation and removal following treatment of L1210 cells with nitrogen mustards," Cancer Communications, vol. 2, no. 12, pp. 387-394, 1990.

[53] M. R. Middleton and G. P. Margison, "Improvement of chemotherapy efficacy by inactivation of a DNA-repair pathway," Lancet Oncology, vol. 4, no. 1, pp. 37-44, 2003.

[54] L. H. Thompson and J. M. Hinz, "Cellular and molecular consequences of defective Fanconi anemia proteins in replication-coupled DNA repair: mechanistic insights," Mutation Research, vol. 668, no. 1-2, pp. 54-72, 2009.

[55] K. Yamamoto, M. Ishiai, N. Matsushita et al., "Fanconi anemia FANCG protein in mitigating radiation- and enzyme-induced DNA double-strand breaks by homologous recombination in vertebrate cells," Molecular and Cellular Biology, vol. 23, no. 15, pp. 5421-5430, 2003.

[56] K. Nakanishi, Y.-G. Yang, A. J. Pierce et al., "Human Fanconi anemia monoubiquitination pathway promotes homologous DNA repair," Proceedings of the National Academy of Sciences of the United States of America, vol. 102, no. 4, pp. 1110-1115, 2005.

[57] A. R. Meetei, A. L. Medhurst, C. Ling et al., "A human ortholog of archaeal DNA repair protein Hef is defective in Fanconi anemia complementation group M," Nature Genetics, vol. 37, no. 9, pp. 958-963, 2005.

[58] A. Ciccia, C. Ling, R. Coulthard et al., "Identification of FAAP24, a Fanconi Anemia Core Complex Protein that Interacts with FANCM," Molecular Cell, vol. 25, no. 3, pp. 331343, 2007.

[59] Y. Masuda, M. Ohmae, K. Masuda, and K. Kamiya, "Structure and enzymatic properties of a stable complex of the human REV1 and REV7 proteins," The Journal of Biological Chemistry, vol. 278, no. 14, pp. 12356-12360, 2003.

[60] M. L. G. Dronkert and R. Kanaar, "Repair of DNA interstrand cross-links," Mutation Research, vol. 486, no. 4, pp. 217-247, 2001.

[61] M. E. Hegi, A.-C. Diserens, T. Gorlia et al., "MGMT gene silencing and benefit from temozolomide in glioblastoma," The New England Journal of Medicine, vol. 352, no. 10, pp. 9971003, 2005.

[62] M. Esteller, J. Garcia-Foncillas, E. Andion et al., "Inactivation of the DNA-repair gene MGMT and the clinical response of gliomas to alkylating agents," The New England Journal of Medicine, vol. 343, no. 13, pp. 1350-1354, 2000.

[63] T. Helleday, E. Petermann, C. Lundin, B. Hodgson, and R. A. Sharma, "DNA repair pathways as targets for cancer therapy," Nature Reviews Cancer, vol. 8, no. 3, pp. 193-204, 2008.

[64] J. A. Quinn, A. Desjardins, J. Weingart et al., "Phase I trial of temozolomide plus $\mathrm{O}^{6}$-benzylguanine for patients with recurrent or progressive malignant glioma," Journal of Clinical Oncology, vol. 23, no. 28, pp. 7178-7187, 2005. 
[65] O. Khan and M. R. Middleton, "The therapeutic potential of $O^{6}$-alkylguanine DNA alkyltransferase inhibitors," Expert Opinion on Investigational Drugs, vol. 16, no. 10, pp. 15731584, 2007.

[66] A. Giese, T. Kucinski, U. Knopp et al., "Pattern of recurrence following local chemotherapy with biodegradable carmustine (BCNU) implants in patients with glioblastoma," Journal of Neuro-Oncology, vol. 66, no. 3, pp. 351-360, 2004. 

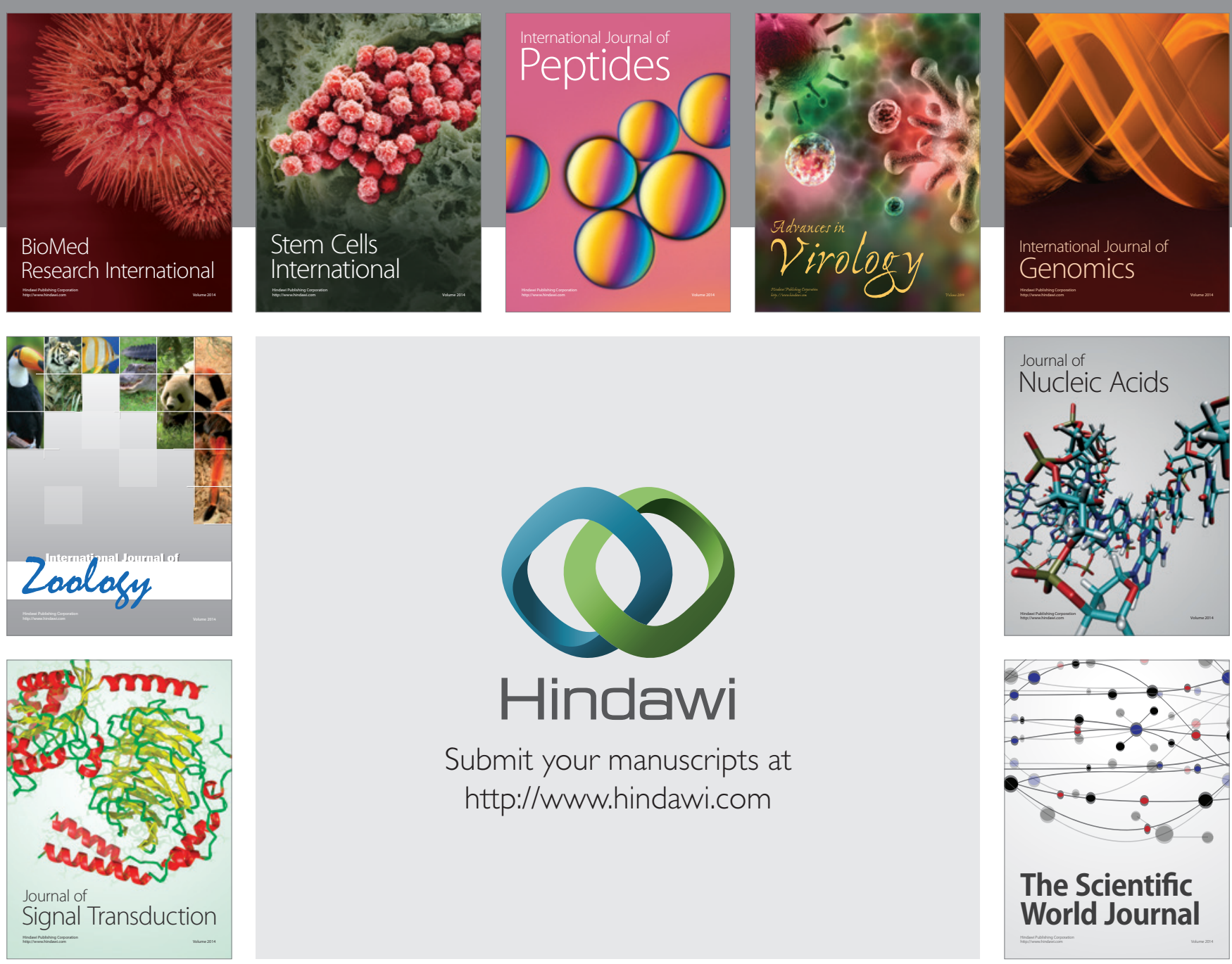

Submit your manuscripts at

http://www.hindawi.com
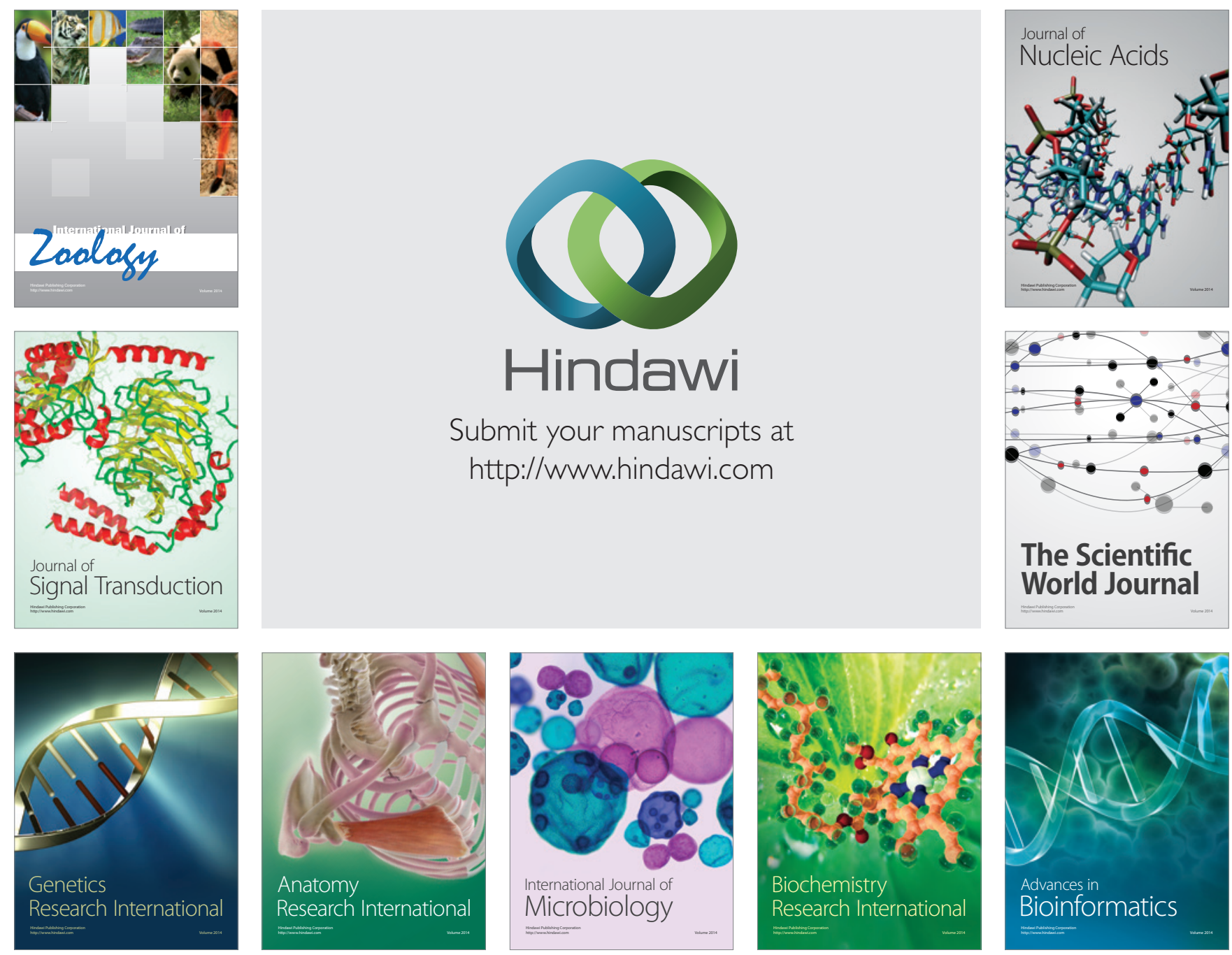

The Scientific World Journal
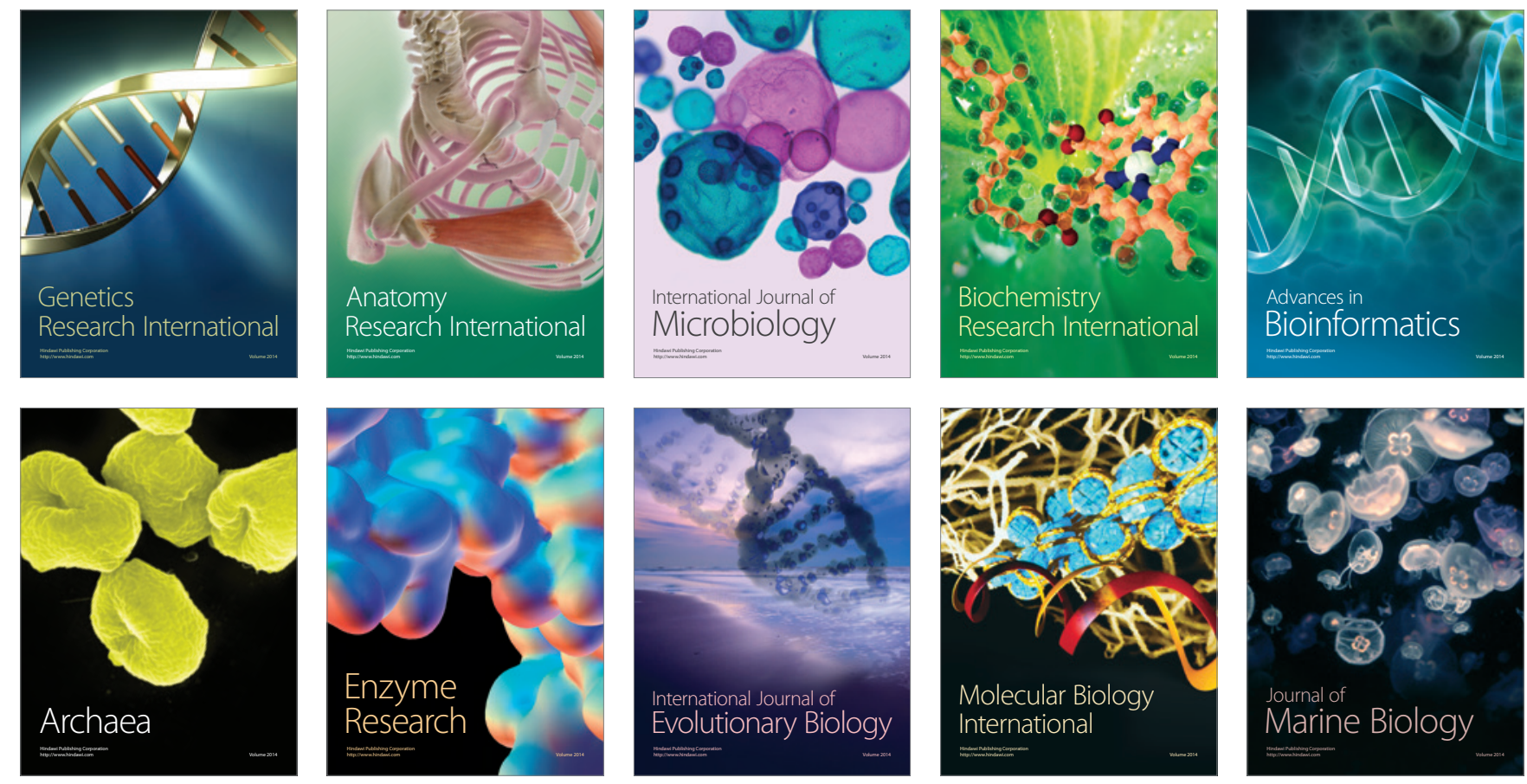